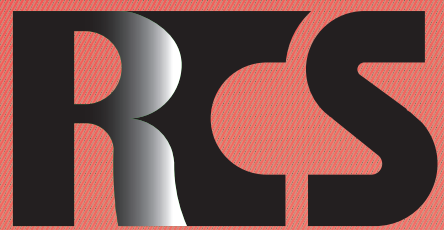

Depósito legal ppi $201502 Z U 4662$

Esta publicación científica en formato digital es continuidad de la revista impresa Depósito Legal: pp $197402 Z U 789$

- ISSN: 1315-9518 • ISSN-E: 2477-9431

Revista de Ciencias Sociales

Universidad del Zulia. Revista de la Facultad de Ciencias Económicas y Sociales Vol. XXVII. No. 2

Abril-Junio 2021

Esta publicación científica en formato digital es continuidad de la revista impresa Depósito Legal: pp $197402 Z$ Z789 ISSN: 1315-9518 

Mendoza-Torres, G., y Villavicencio-Aguilar, C. (2021). Tradición pesquera artesanal e identidad sociocultural de Puerto Bolívar: Contexto del Golfo de Guayaquil-Ecuador. Revista de Ciencias Sociales (Ve), XXVII(2), 386-400.

\title{
Tradición pesquera artesanal e identidad sociocultural de Puerto Bolívar: Contexto del Golfo de Guayaquil-Ecuador
}

\author{
Fernández-Espinosa, Cira* \\ Brito-Paredes, Patricio** \\ Mendoza-Torres, Galo ${ }^{* * *}$ \\ Villavicencio-Aguilar, Carmita*****
}

\section{Resumen}

La pesca es tenida como una de las principales actividades de subsistencia en el mundo entero y una de las maneras más antiguas mediante la cual las personas han resuelto parte de sus necesidades básicas. En el caso de la pesca artesanal, además de ser una fuente de alimentación, también resulta una opción importante para la obtención de ingresos económicos y, más aún, para la generación de vínculos sociales entre quienes se dedican a ella. Esta es la realidad que se muestra, concretamente, las realidades sociales, culturales y económicas que rodean a la pesca artesanal como tradición y generadora de identidad sociocultural en el contexto de Puerto Bolívar, en el Golfo de Guayaquil. La investigación tiene como objetivo analizar la relación entre tradición pesquera e identidad cultural desde la hermenéutica de diversos textos y fuentes institucionales que han sido contrastadas con la realidad histórica y geográfica en la zona. Entre los resultados destacan el arraigo más cultural que económico de la pesca; su afectación económica y cultural, además de daños ecológicos por la tala de manglares para construir camaroneras. Se concluye, que para el diseño y ejecución de políticas públicas, se debe respetar las prácticas culturales y el tema ecológico.

Palabras clave: Pesca artesanal; actividades de subsistencia; tradición; identidad sociocultural; Puerto Bolívar-Ecuador.

Doctora en Ciencias, Filosofía. PhD. en Ciencias Sociales y Humanismo. Máster en Planificación y Administración Educativa. Licenciada en Ciencias de la Educación. Profesora Principal de la Universidad Técnica de Machala, Ecuador. E-mail: cfernandez@utmachala.edu.ec iD ORCID: http://orcid.org/0000-0002-1483-2639

** Máster en Administración y Planeamiento Educativo. Economista Docente Titular e Investigador de la Universidad Técnica de Machala, Ecuador. E-mail: mbrito@utmachala.edu.ec (iD ORCID: http://orcid.org/0000-0001-7042-5547

*** Máster en Docencia Universitaria e Investigación Educativa. Licenciado en Sociología y Ciencias Políticas. Sociólogo. Docente Investigador de la Universidad Técnica de Machala, Ecuador. E-mail: gmendoza@utmachala. edu.ec iD ORCID: http://orcid.org/0000-0003-3687-6875

***** Doctora Psicorehabilitadora y Educadora Especial. PhD. en Desarrollo Psicológico, Aprendizaje y Salud. Magíster en Educación Especial. Profesora Titular en la Universidad Técnica de Machala, Ecuador. E-mail: cvllavicencio@ utmachala.edu.ec (iD ORCID: http://orcid.org/0000-0003-2614-7062 


\title{
Artisanal fishing tradition and sociocultural identity of Puerto Bolívar: Context of the Gulf of Guayaquil-Ecuador
}

\begin{abstract}
Fishing is considered one of the main subsistence activities throughout the world and one of the oldest ways in which people have met part of their basic needs. In the case of artisanal fishing, in addition to being a source of food, it is also an important option for obtaining economic income and, even more, for generating social ties between those who engage in it. This is the reality that is shown, specifically, the social, cultural and economic realities that surround artisanal fishing as a tradition and generator of sociocultural identity in the context of Puerto Bolívar, in the Gulf of Guayaquil. The research aims to analyze the relationship between fishing tradition and cultural identity from the hermeneutics of various texts and institutional sources that have been contrasted with the historical and geographical reality in the area. Among the results are the more cultural than economic roots of fishing; its economic and cultural impact, as well as ecological damage due to the cutting down of mangroves to build shrimp farms. It is concluded that for the design and execution of public policies, cultural practices and the ecological issue must be respected.
\end{abstract}

Keywords: Artisanal fishing; subsistence activities; tradition; sociocultural identity, Puerto Bolívar-Ecuador.

\section{Introducción}

Una de las actividades económicas más antiguas en el mundo está referida a la pesca, especialmente, aquella que se realiza de manera artesanal. De hecho, se puede considerar que las fuentes hídricas, particularmente las marítimas han funcionado históricamente como uno de los grandes centros de producción natural de alimentos, no solo de fácil acceso, sino con la cualidad de ser una importante fuente de proteínas (GonzálezGarcés, 2006).

Originalmente, la pesca fue una actividad realizada como forma de subsistencia, en tal sentido, puede decirse que tuvo un origen artesanal. No obstante, en su evolución es considerada pesca artesanal, aquella que -aunque se haga con fines de comercialización-, su nivel de impacto ambiental es casi nulo y tampoco tiene la finalidad de industrializar el producto. Según la Organización de las Naciones Unidas para la Alimentación y la Agricultura (FAO, 2003), es capaz de generar empleos, al tiempo que requiere mucha menos inversión, tanto en el tipo de embarcaciones que se utilizan como en la cantidad de combustible que se gasta. En definitiva, este tipo de pesca abarca toda aquella que se realiza en forma manual y puede ser rudimentaria, solo de subsistencia o con ciertos fines comerciales.

Hay que considerar que, a diferencia de las bondades naturales de la pesca artesanal, debido a sus métodos selectivos, la pesca industrial es altamente perjudicial para muchas especies que no son su objetivo principal, pero que son afectadas incidentalmente. Ese impacto, se siente tanto en el ambiente como en aquellos pescadores que se desempeñan en el campo artesanal, cuyas principales presas son destruidas por la forma de pesca intensiva $\mathrm{y}$ de arrastre, que ejecutan las personas o empresas que se dedican a la industrialización del producto pesquero, buscando básicamente, una fuente de lucro.

En el caso ecuatoriano, el análisis de la pesca artesanal pasa por reafirmar su 
Fernández-Espinosa, Cira; Brito-Paredes, Patricio; Mendoza-Torres, Galo y VillavicencioAguilar, Carmita

Tradición pesquera artesanal e identidad sociocultural de Puerto Bolivar: Contexto del Golfo de Guayaquil-Ecuador

importancia para la dinámica económica, siendo, conjuntamente con el petróleo, uno de los productos más importantes del país. La misma FAO (2019); así como Álvarez, et al. (2020), señalan que en Ecuador, la industrialización del producto pesquero es una consecuencia lógica del crecimiento del sector, la ampliación de sus redes de comercialización $\mathrm{y}$, por supuesto, la especialización de los trabajadores para optimizar sus métodos $\mathrm{y}$ obtener mayores recursos y calidad de vida.

Las características socioeconómicas del país, incluyen una importante franja de población, sobre todo habitantes de la Costa ecuatoriana, dedicada -casi exclusivamente-, a la pesca como actividad económica y de subsistencia. En su versión artesanal, la actividad pesquera tiene una larga historia en la zona, especialmente por la riqueza de peses que posee el Golfo de Guayaquil. Allí, fueron surgiendo paulatinamente una serie de puertos que permitieron una consolidación de la pesquería artesanal, aunque mantuvo sus características básicas como actividad no industrial.

En esa perspectiva, la pesca artesanal es parte de esa dinámica natural en la que las personas, particularmente los latinoamericanos, comprenden el desarrollo como una acción, no de explotación de recursos y lucro, sino como un equilibrio entre la humanidad y la naturaleza. Avanzar en esa forma de comprender el desarrollo y combatir la pobreza, pasa por superar esas nociones economicistas de esta última que coloca el énfasis en las posesiones de bienes (Alvarado, 2016; Anaya, Buelvas y Romero, 2020).

Partiendo de estas convicciones, se ha planteado una aproximación hermenéutica a lo que representa la actividad pesquera en Puerto Bolívar, con la intención de develar los vínculos socioculturales y económicos que existen entre esa práctica y la construcción de la identidad cultural de los habitantes de esta zona. De lo que se trata, es de visualizar la pesquería artesanal, no solo como una forma que tienen los pobladores de esta comunidad de resolver su sustento diario, sino de encontrar en ella lo que no aparece a simple vista, aquello que representa como vinculo social y que le convierte en una fuente de identidad de los pescadores.

Concretamente, la intención de los autores como objetivo de investigación es analizar la relación entre tradición pesquera e identidad cultural desde la hermenéutica de diversos textos y fuentes institucionales, que han sido contrastadas con la realidad histórica y geográfica en la zona. Así, puede construirse una imagen más cercana y actualizada de una de las actividades económicas ancestrales de mayor significación cultural en el país y visualizar sus perspectivas de cara a este, aún naciente siglo XXI.

\section{Algunas orientaciones teóricas respecto a la pesca artesanal como tradición}

La pesca artesanal, es una actividad tradicional con su propia impronta sociocultural, un legado a posterioridad que se reproduce generacionalmente como una práctica sociofamiliar, que se crea y recrea desde la cotidianidad como manifestación de la cultura popular. "La pesca es una de las actividades primigenias efectuadas por el hombre para su subsistencia alimentaria, economía y cohesión social" (León-Valle, et al., 2017, p.20).

Los pescadores artesanales, mantienen así, sus costumbres, tradiciones, fiestas, rituales religiosos, gastronomía y demás manifestaciones propias, que son parte intrínseca de su identidad cultural. Sin embargo, a pesar de los agresivos procesos de aculturación impulsados desde las nuevas tecnologías de la información y la comunicación, así como la afectación de la actividad por el desarrollo de artes sofisticadas de pesca, sigue sobreviviendo como mecanismo de protección social de una herencia generacional de los saberes ancestrales (León-Valle, et al., 2017).

En este sentido, las tradiciones de la pesca artesanal más utilizadas en las caletas 
pesqueras, se estructuran alrededor de la utilización de instrumentos tales como: El espinel de fondo o línea de fondo, línea de superficie (artes que utilizan el anzuelo); chinchorro o cerco, atarraya, enmalle, mallón, bolso y el trasmallo (artes que utilizan redes).

Las más usadas son la línea de mano, palangres y espineles. La primera, para la pesca de picudo, dorado, la corvina, bonito, trompeta y bagre. Algunos pescadores prefieren el espinel, por no dejar marcas en el producto y ser mejor para la exportación (León-Valle, 2017). Todas estas artes son amigables con el medio ambiente. El trasmallo corvinero, utilizado en faenas pesqueras próximas a la costa, capturan cachemba, lisa, carita, bagre, Leonor, sano, róbalo, pámpano, berruguete y camarones.

Más aún, las artes de pesca, para continuar siendo de carácter ancestral y preservar el estado del recurso y el bienestar de las comunidades pesqueras que dependen de él, demandan una pesca responsable y ordenada (Working to sustain the natural world for the benefit of people and nature [WWF-Laos], 2016), exigen el cumplimiento de ciertas condiciones y regulaciones, con la decisión y determinación moral que imponga la comunidad, además de mantener la utilización de las artes manuales de pesca y el uso de embarcaciones.

Se deja ver en cada una de estas regulaciones y aspiraciones institucionales, sobre la actividad pesquera artesanal, la importancia que este sector tiene, no solo vista desde su perfil económico, sino en su significación simbólica cultural. Cada modalidad, cada método para la pesca, no es más que una muestra del ingenio y la creatividad humana, que está determinada, en este caso, por el contexto geotemporal donde se sitúa. Por supuesto, sigue planteado ese forcejeo, tanto a nivel de lo simbólico, como de la praxis, entre la industrialización y la tradición, porque eso es parte de la complejidad con la que avanzan las sociedades.

\section{Caracterización del contexto: Puerto Bolívar en el Golfo de Guayaquil}

El Golfo de Guayaquil, es un estuario de gran dimensión ubicado en la costa sudamericana del Pacífico, se extiende en 200 $\mathrm{Km}$., de norte a sur, desde la Puntilla de Santa Elena en Ecuador hasta cerca de Máncora en Perú y penetra en el interior del litoral ecuatoriano a una distancia de $120 \mathrm{Km}$. Ubicar Puerto Bolívar es situar a la isla Puná con sus tres canales: Morro, Cascajal y Jambelí, en este último se encuentra un Archipiélago y su isla con su mismo nombre. Puerto Bolívar, está localizado al borde este del estero Santa Rosa frente a la Isla Jambelí. Existen ocho principales puertos de desembarque artesanal de Ecuador: Esmeraldas, Manta, San Mateo, Santa Rosa, Anconcito, Engabao, Playas y Puerto Bolívar; los cuatro últimos pertenecen al Golfo de Guayaquil (Herdson, Rodríguez y Martínez, 1985).

La costa Sur del Ecuador, que comprende el perfil de la Provincia de El Oro, es un sector oceanográfico muy rico por cuanto la baña el Océano Pacífico y la corriente cálida del Niño, en este espacio hay encuentros culturales entre el pescador ecuatoriano y el del norte del Perú, se conoce que desde la Colonia y mucho antes los pescadores viajaban desde Puná, por la costa de El Oro, las islas de Jambelí, hasta el Puerto de Paita, para intercambiar productos así como mercancías y, en ese contacto, compartían conocimientos y diversas costumbres. La comunidad de pescadores de Puerto Bolívar es significativa, la más grande de la provincia de El Oro, tal como se muestra en la Figura I. 
Fernández-Espinosa, Cira; Brito-Paredes, Patricio; Mendoza-Torres, Galo y VillavicencioAguilar, Carmita

Tradición pesquera artesanal e identidad sociocultural de Puerto Bolivar: Contexto del Golfo de Guayaquil-Ecuador

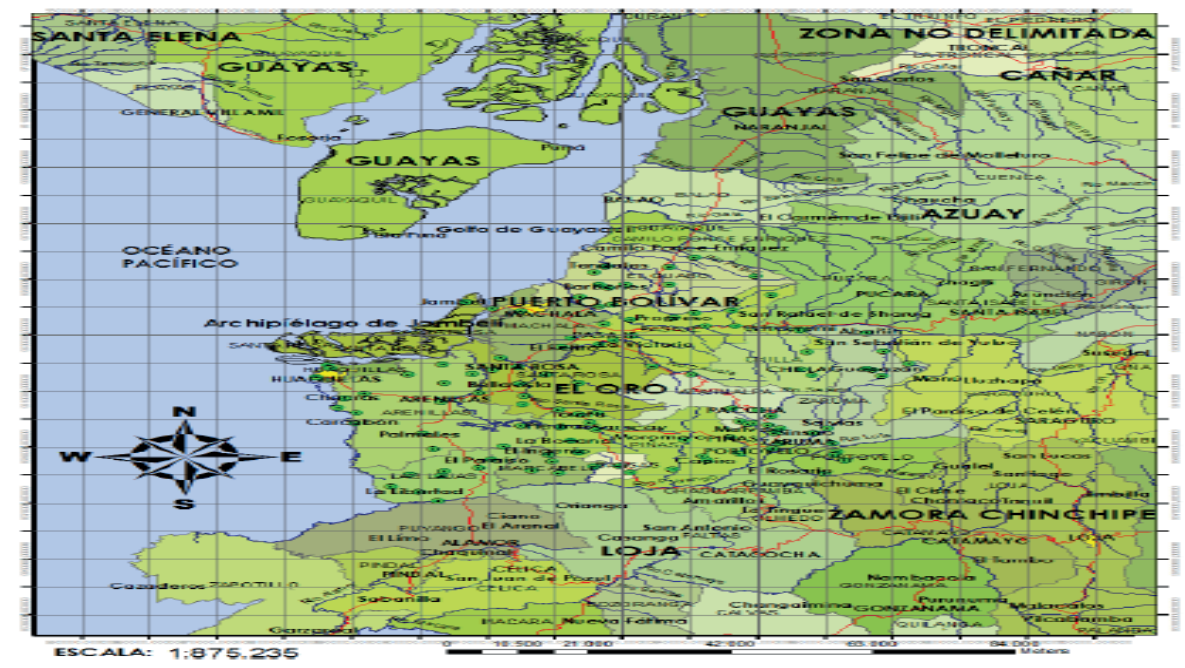

Fuente: Aguilar (2013).

Figura I: Ubicación geográfica de Puerto Bolívar

En definitiva, aunque se trata de un contexto, geográficamente extenso, considerando que el Golfo también es parte del Perú, la zona en la que se ubica esta investigación, mantiene ciertos rasgos de homogeneidad respecto a la actividad de la pesquería artesanal. En el análisis, hay elementos que pueden asociarse con las formas procedimentales y de pensamiento autóctono de la región, que permiten tener una visión integral de las características sociales, económicas y, mejor aún, culturales de este contexto.

\section{Caracterización histórico-temporal de la pesca artesanal en Puerto Bolívar}

El Programa de Manejo de Recursos Costeros (PMRC, 1993), señala que el borde costero de Puerto Bolívar, hasta mediados de los años 50, estuvo cubierto de un gran bosque de manglar con árboles que tenían una altura de hasta 10 metros, por entre los cuales se abrían paso esteros y canales y, en esos años, eran sitios de abundante recolección de conchas, cangrejos, jaibas, mejillones y ostiones. Así como lugar de anidación de muchas aves marinas; aunque -con el pasar de los años, y con el advenimiento de la actividad bananera para la exportación a finales de 1950 y la actividad camaronera- este panorama natural cambió radicalmente.

En la década de los años 70, el manglar fue desapareciendo progresivamente: $\mathrm{Al}$ principio, por la ocupación de suelos con fines habitacionales para los migrantes y construcciones portuarias (Machala y Puerto Bolívar) y luego por la construcción de piscinas para el cultivo del camarón en cautiverio, donde -esta última- es la que desgastó en corto tiempo los manglares.

Por esta época, el borde costero llegó a constituirse en un polo de desarrollo económico y un lugar de atracción para inmigrantes nacionales, especialmente de las provincias de Esmeraldas, Manabí y 
Guayas (Aguilar, 2013). Además, se destaca la participación de ciudadanos de la sierra, principalmente de las provincias de Azuay y Loja. Con la tala indiscriminada del manglar ha habido destrucción de su ecosistema, se han contaminado los estuarios, alterado cursos naturales de agua y atentando contra las personas que viven en las zonas costeras que dependen directamente de la biodiversidad del lugar y de la producción de los pescadores (Gobierno Autónomo Descentralizado [GAD] de Machala, 2018).

El manglar fue y es la fuente de captura y recolección de pesquerías artesanales: $\mathrm{La}$ actividad siempre ha subsistido por la presencia de este bosque, siendo la siguiente secuencia temporal la forma como se han utilizado los bosques de manglar en Puerto Bolívar

\subsection{La situación de la costa antes de 1971}

Hasta comienzo de esta década, la descripción general de la zona incluía: a) Un hábitat de moluscos, crustáceos y peces; b) la producción de madera para construcción de casas, artes de pesca, carbón - leña; c) la producción de corteza para obtener taninos; y, d) una conversión de áreas de manglar en centros poblados (PMRC, 1993). Este es además, un periodo importante para la evolución económica de la región, impulsada de manera decisiva, por la industria bananera, aunque, por esa misma razón, se produjeron una serie de afectaciones ecológicas y problemas en el abasto de agua.

En esta época, es común que se haya subvalorado la importancia de las costas ecuatorianas, pues igualmente ocurrió en muchos países de América Latina. Fue precisamente, esa situación de depresión social y económica, la que, inicialmente presionó para la diversificación de las fuentes de ingresos de las poblaciones allí asentadas. Es en este período donde comienza a cimentarse un manejo integral de los recursos con los que se cuenta, al tiempo que se socializan ciertos valores y toma de conciencia acerca de la necesidad de cuidar esos recursos.

\subsection{La situación en entre 1971-1991}

Para estas dos décadas hay cambios importantes, entre los cuales se cuenta con: a) La conversión del manglar en áreas portuarias; b) conversión del manglar en camaronera; c) producción de madera para construcciones rústicas; d) zonas de pesca de larvas de camarón; y, e) sitios de recolección de mariscos (PMRC, 1993). En este periodo, también continúa siendo importante la producción bananera, por lo que se da una especie de diversificación económica que contribuye a un paulatino mejoramiento de las condiciones materiales de existencia de la población.

Desde el punto de vista cultural, la actividad pesquera tradicional se ve impactada por el auge de la industria camaronera, que trae consigo un conjunto de nuevas prácticas socioeconómicas que tienen su correlato en el ámbito de lo simbólico. Las nuevas generaciones, ven allí una mejor y más rápida fuente de recursos, soslayando el impacto que esta actividad tiene sobre los manglares y otras riquezas naturales. De cualquier manera, desde la lógica economicista, se puede decir que hay un auge económico en la región, consolidada alrededor de sus principales recursos.

En la zona, las comunidades de pescadores artesanales, siempre han reconocido la importancia de los manglares para su supervivencia, pero no han tenido poder político ni económico para ejecutar y desarrollar actividades de conservación y protección de este ecosistema. Las leyes existentes son poco o nada acatadas por los inversionistas, cuya meta es lograr mayor rentabilidad en sus negocios, para ello, convertir áreas de manglar a camaroneras es una actividad altamente lucrativa.

El Gobierno Nacional, ha desplegado una serie de convenios con los pescadores artesanales. Es así como, el Ministerio de Acuaculturay Pesca(2017)entregóconcesiones de áreas para recolección de cangrejos. Así mismo, El Ministerio del Ambiente y 
Fernández-Espinosa, Cira; Brito-Paredes, Patricio; Mendoza-Torres, Galo y VillavicencioAguilar, Carmita

Tradición pesquera artesanal e identidad sociocultural de Puerto Bolivar: Contexto del Golfo de Guayaquil-Ecuador

Agua (2016), traspasó 900 hectáreas para conservación de los manglares a "Guardianes del Manglar", tras la firma del Acuerdo de Uso Sustentable y Custodia de Manglar, realizada entre el Ministerio del Ambiente y Agua con la Asociación de Producción Pesquera Artesanal (ASOCHUGRA), con el compromiso y corresponsabilidad de cuidar la especie y respetar las medidas de captura en este escenario de gran biodiversidad y con más seguridad durante sus faenas de pesca.

Como ha podido observarse, cuando se trata de caracterizar la pesca artesanal en Puerto Bolivar, inmediatamente surge la complejidad sociocultural como un rasgo determinante. Tanto la situación de los pescadores, en sus dilemas para correlacionar sus tradiciones con el oficio que les permite sobrevivir, como el desarrollo de las poblaciones y de las instituciones involuradas en el fenómeno de la pesquería, tienen que ser incluidas en el foco de atención de cualquier estudio serio que se haga sobre este tema, pues se cree que esta será la única forma en que, las políticas públicas pueden ser asertivas para coadyuvar en el mejoramiento de las condiciones de vida (McGoodwin, 2002) de las personas que habitan esta región y que practican la pesca artesanal.

\section{Otras caracterizaciones geo- temporales de la actividad pesquera en el contexto de Puerto Bolívar}

La pesca artesanal, en la provincia de El Oro en general y en Puerto Bolívar en particular, es una actividad ancestral que se ha desarrollado como una acción de subsistencia con implementos y embarcaciones sencillas, hasta barcas modernas construidas con fibra de vidrio (Herrera, et al., 2013).

Así, la actividad de la pesca como la recolección de conchas, son oficios que han pasado de generación en generación, han heredado el oficio de sus padres y abuelos (El Comercio, 2009), y la actividad ha trascendido durante muchos años, al punto que cerca del
$60 \%$ de los pescadores artesanales orenses están ubicados en Puerto Bolívar. En algunas familias va desapareciendo paulatinamente, en otras, son consideradas tradiciones que no deben desaparecer; no obstante, el criterio actual de muchos mariscadores es que el "ser conchero no es un oficio que produzca mucho dinero, pues la concha no siempre es pagada a buen precio" (E1 Telegráfo, 2014, p.1).

El Gobierno Autónomo Descentralizado (GAD) de Machala, en el Plan de Desarrollo y Ordenamiento Territorial, informó sobre las condiciones estuarinas del filo costero orense. Esta zona siempre estuvo favorecida por la presencia de una amplia variedad de especies marinas, cuya pesca y comercialización han venido contribuyendo al desarrollo socioeconómico de las comunidades de pescadores y mariscadores que habitan en el sector. El desarrollo de estas actividades, se ha visto beneficiado y fortalecido desde épocas ancestrales por la presencia del manglar que es uno de los ecosistemas más ricos en biodiversidad, cuya conservación es vital para la sustentabilidad de los recursos estuarinos (GAD, 2018).

El esfuerzo pesquero en Puerto Bolívar es estacional y se efectúa en pesca de altura y de peces demersales (aguas profundas). Los productos del mar que son parte de la pesca artesanal en todo el Golfo y, por ende, en Puerto Bolívar, son precriaderos artesanales de camarones, concha prieta, camarón, langosta, pepino de mar, cangrejo rojo, peces pelágicos grandes (dorados, picudos) y gran densidad de peces demersales, como corvina, robalo, pargo, cherna, cachema, que son recogidos en el fondo del mar, a 200 metros de profundidad de la plataforma continental; los pescadores de este puerto utilizan una gran variedad de redes de enmalle (Paredes, 2000).

La captura de cangrejos en el Golfo de Guayaquil es principalmente manual: Atrapan machos y hembras que están a menos de un metro de profundidad. En Puerto Bolívar los cangrejeros migrantes de Manabí trajeron consigo la captura con gancho. Los pescadores locales la han adoptado por ser una técnica eficiente. 
Según el Instituto Nacional de Pesca (INP, 2019), esta actividad de acopio era más realizada por mujeres y niños; con el trascurso del tiempo, por la contaminación del manglar optaron por otro emprendimiento como es la elaboración de artesanías con restos de conchas, escamas y huesos, para ello fueron concedidas a la Asociación de Mujeres de Puerto Bolívar 140 hectáreas de manglar del Estero de Huaylá para su conservación y fuente de recursos reutilizables.

De cualquier modo, las condiciones en las que ha evolucionado y se mantiene la actividad pesquera en Puerto Bolívar, conservan un equilibrio entre la importancia que sus pobladores le otorgan a la pesca, como tradición, y el significado económico, que la misma tiene al momento de evaluar su peso como parte de la dinámica de subsistencia de las familias. Lo que ha podido observarse es que, a pesar de lo cíclico que puede ser el oficio de la pesca artesanal, tanto el Estado ecuatoriano, como las organizaciones familiares y empresariales de pequeña escala, siguen enfocados en mantener su idoneidad ambiental y económica.

\section{Metodología}

La metodología bajo la cual se produce este estudio, corresponde a una investigación descriptiva - exploratoria, con una técnica documental en la que se utilizó una búsqueda sistemática de información en plataformas académicas reconocidas, tales como: Scielo, Redalyc, Dialnet, Scopus, entre otras. A partir de allí, se seleccionaron algunos textos con información relevante sobre el tema, colocando en práctica los criterios de inclusión referidos a pertinencia, calidad, actualidad y exhaustividad.

Asimismo, hay elementos de la observación cualitativa puestos en práctica para contrastar la teoría encontrada en los documentos, con la realidad concreta que muestra el contexto en el que se circunscribe este artículo. La observación, se realizó en el Golfo de Guayaquil, considerando la actividad pesquera artesanal que allí se lleva a cabo y los elementos socioculturales que involucra. Hay que aclarar, que fue una observación de carácter informal, pero que cubre el elemento histórico del tema y la presencia actual de sus elementos más importantes en la cotidianidad de las personas, quienes son finalmente, las que le dan contenido a los resultados de la indagación.

En ese orden de ideas, conviene aclarar que, siendo este un estudio teórico, desde la introducción hasta las conclusiones, se plasman los resultados de una indagación exhaustiva de la documentación producida sobre el tema de la pesca artesanal, su historia y sus vinculaciones específicas en la construcción de la identidad sociocultural de pueblos como Puerto Bolívar. Se puede agregar entonces, que en la fase hermenéutica se construyeron algunos elementos teóricos-conceptuales que describen, con las precisiones que permiten este tipo de estudios, las realidades que definen las relaciones entre las actividades económicas de la pesca artesanal y los rasgos sociales y culturales, que se van articulando así como entrelazando con esas actividades, las cuales, tienen una perspectiva que supera lo estrictamente económico.

\section{Resultados y discusión}

\subsection{Respecto a la identidad sociocultural}

Entre los aspectos cualitativos más interesantes de la realidad observada, se encuentran aquellos de carácter social y cultural, que conforman la identidad y las representaciones sociales de los pescadores de la comunidad de Puerto Bolívar. Su lenguaje, sus formas de socializar, sus aspiraciones y emociones, que se manifiestan como la herencia cultural de los adultos (ancianos en la mayoría de los casos); paulatinamente transformadas por las costumbres de migrantes de la parte alta de la provincia de El Oro, de Guayas y Esmeraldas, que llegan a trabajar en 
Fernández-Espinosa, Cira; Brito-Paredes, Patricio; Mendoza-Torres, Galo y VillavicencioAguilar, Carmita

Tradición pesquera artesanal e identidad sociocultural de Puerto Bolivar: Contexto del Golfo de Guayaquil-Ecuador

las actividades relacionadas con el embarque del banano. La categoría representación social, es importante para explicar cómo los pescadores se ven en el contexto de sus actividades y relaciones.

En ese sentido, los pescadores establecen relaciones con el contexto ambiental y con los protagonistas de la actividad pesquera. Méndez (2020), plantea que la representación social es el medio para evidenciar estos procesos:

Por un lado, esta categoría permite evidenciar las relaciones que los pescadores artesanales establecen con el ambiente (río, isla, recurso pesquero) en el espacio. Por otro lado, desarrollan "prácticas territoriales" en la interrelación con otros actores como el Estado y las empresas privadas que pueden estar influidas por distintas representaciones sobre el territorio. (p.198)

A los asentamientos de los pescadores, se les denomina caletas, en ellas se adquiere y representa la identidad de los pueblos en su cultura y toda la expresión de la cotidianidad así como de la vida. Es la unidad geopolítica administrativa y el centro de todo tipo de actividad; en este espacio se realiza la faena pesquera y se intercambian saberes: La época de pesca, estaciones, los vientos, las corrientes, ubicación, espacio, cuándo, cómo y dónde pescar, cómo confeccionar sus redes, materiales de pesca, el tipo de carnada para cada especie, cómo se construye o diseña las artes de pesca, ubicación de las especies; el tipo de especies demersales o pelágicas, sus hábitos, el movimiento del cardumen, la velocidad de traslado, que convierte a los pescadores en sabios de las artes pesqueras.

Estas prácticas forman un constructo social, de un saber diario que se consolida en el ejercicio de la cotidianidad, de manera que la actividad pesquera en las caletas ha sido una especie de cohesionador social y el centro de las actividades comunitarias. "La sostenibilidad de cualquier sociedad dependerá en gran medida del grado de diversidad y autoconfianza que pueda mantener con respecto a la reproducción de sus características sociales y culturales" (Kurien, 2020, p.206).

La identidad que es calificativo de "nosotros"; y, el "yo", frente a los "otros", se construye en la práctica diaria, en el compartir las actividades con amigos, allegados, familiares y la comunidad; en la dialéctica de las interacciones con los elementos de la naturaleza, del mar, del clima y sus rutinas cotidianas, que finalmente, contornean las características particulares de los pobladores de la costa.

Los pescadores artesanales -en otras épocas- navegaban en sus canoas, elaboradas en el puerto, actualmente, lo hacen en botes modernos de fibra. Al llegar a este puerto artesanal, existen unos 50 atracaderos, donde las embarcaciones cargan el hielo y desembarcan las capturas (Leante y García, 2012). Asimismo, suelen levantarse con los albores del día y regresar al anochecer; y, otras veces, entre dos y tres días después, en dependencia del lugar donde realizan la pesca. A su regreso, sea por la playa o por muelles pequeños, los esperan los comerciantes intermediarios, ellos revisan las piezas e imponen el precio; discuten, pero llegan a acuerdos. "Las prácticas territoriales relacionadas con las actividades productivas que realizan cada uno de los diferentes actores, configuran relaciones de poder desiguales entre ellos y con el territorio" (Méndez, 2020, p.15).

Las relaciones de poder, en el mar y en tierra son diferentes, en la primera, se impregnan de valor, coraje, atrevimiento; pero, en tierra, se entra en negociaciones para establecer convenios importantes y así no perder su pesca: El proceso pasa por momentos traumáticos, puesto que en ocasiones no se da un justo valor al trabajo que el pescador artesanal realiza en el mar.

Los recolectores de conchas y cangrejos, tienen importancia significativa en el desembarque de su producto, pero ahora están disminuidos por el establecimiento de camaroneras y la pérdida de los manglares. En esta zona de pesca, han existido múltiples desavenencias entre la pesca industrial y la pesca artesanal. Esta situación ha provocado más de un problema, como el suscitado el 15 de agosto de 2018, en el que los pescadores 
artesanales protestaron, manifestaron su inconformidad por la situación que vivían, con desmanes y violencia (fue necesaria la intervención de la fuerza pública para controlar esta manifestación).

La prensa local detalló este acontecimiento: "Según argumentan sus dirigentes, la escasez de especies está relacionada con la incursión de embarcaciones de pesca industrial en las 8 millas náuticas. Los acuerdos ministeriales 2305 y 080 establecen que esa área es exclusiva para la pesca artesanal" (El Comercio, 2018, p.1). Según el mismo diario, hay más de 84 expedientes administrativos sancionadores a las embarcaciones bolicheras, en lo que va del año. La desconfianza de los pescadores, se evidencia debido al incumplimiento de la guardianía y su ausencia en las costas, que los deja desamparados por la presencia de piratas armados que asaltan las embarcaciones de los pescadores artesanales.

En ese sentido, hay que decir que, a pesar de la importancia que encierra la actividad pesquera artesanal en Puerto Bolívar, existen una serie de limitantes que obstaculizan un mayor impacto económico y social. A la reciedumbre del trabajo, se suma la incertidumbre ante la suerte de captura, el riesgo de asaltos de la delincuencia o piratas, el escaso apoyo gubernamental al sector, los bajos niveles de ingresos obtenidos, la presencia excesiva de intermediarios, la falta de control oficial de precios, el abuso invasivo de la pesca industrial en áreas de uso exclusivo para pesca artesanal y la debilidad organizacional (Banchón, Vite y Carvajal, 2020).

Por su parte, el tema de la religiosidad, también hace presencia constantemente entre sus prácticas cotidianas y es muy característica entre los pescadores. La mayoría bautiza sus botes con el nombre de la Virgen o un santo de su devoción; pese a la religión católica que profesan, su estado civil generalmente es de unión libre, muy pocos tienen parejas a través del matrimonio eclesiástico, esto demuestra de alguna manera que los pobladores han perdido confianza no solamente en las autoridades civiles, sino también en las religiosas, pues el matrimonio "por la Iglesia" casi ha desaparecido.

Desde la perspectiva social y económica, los resultados de este estudio presentan una dependencia total de la mujer y sus hijos, respecto a las figuras masculinas en los hogares. El padre, viene a significar el "jefe", cuando el padre falta, los hijos asumen las responsabilidades laborales. Entre las pocas mujeres que intervienen en las actividades de mar, se encuentran las comerciantes intermediarias o las recolectoras; las mujeres participan activamente en la pesca artesanal, Álvarez, et al. (2017), corroboran este hecho:

La actividad de recolección de orilla, ha sido en el transcurso de la historia de la pesca artesanal, una actividad en la que se han insertado en mayor medida las mujeres, ya sea para la venta de productos, como para el consumo del hogar. (p.30)

Sin embargo, dadas las necesidades económicas actuales, mayor cantidad de mujeres tienden a dedicarse a las actividades que derivan de la pesca artesanal, vinculadas a la venta de los productos directamente $\mathrm{u}$ ofreciendo los mismos para consumo alimenticio en picanterías y restaurantes aledaños al sector del intercambio de la pesca.

Hay factores de violencia intrafamiliar, posiblemente se condicionen por el elevado consumo de drogas y alcohol, a esto se agrega el nivel educativo que es de primaria. Sin ninguna otra formación laboral, la pesca se convierte en una actividad familiar. Los hijos aprenden de los padres y desde jóvenes se incorporan a las actividades pesqueras, que las realizan casi en su totalidad los hombres.

En lo gastronómico, es predominante la alimentación con marisco, pescado y plátano verde. Según opinión de la población turística, la comida porteña es la mejor del país:

En la carta de los locales comerciales, ubicados a lo largo del malecón de esta localidad, preparaciones como el cebiche de camarón o de pescado, majarisco, arroz marinero, sopa marinera, ensalada de cangrejo, corvina frita, camarones apanados y chicharrones de pescado, 
Fernández-Espinosa, Cira; Brito-Paredes, Patricio; Mendoza-Torres, Galo y VillavicencioAguilar, Carmita

Tradición pesquera artesanal e identidad sociocultural de Puerto Bolivar: Contexto del Golfo de Guayaquil-Ecuador

siempre acompañados de verde, se preparan durante toda la jornada para los visitantes que llegan de varias partes del país e incluso del extranjero. (El

Universo, 2018, p.1)

Los porteños son alegres, les gusta evidenciar sus logros económicos, si bien pueden ser desconfiados con las instituciones del Estado, entre ellos son amigos por excelencia, se conduelen del vecino, son solidarios en la desgracia, desafortunadamente, las condiciones socioculturales no apoyan para el desarrollo de un espacio económico importante y que sustente a la población.

\subsection{Respecto a la realidad socioeconómica}

La información oficial del Ministerio de Agricultura y Ganadería de Ecuador (MAG), señala que, en la provincia de El Oro, para el año 2020, existen 11.000 pescadores artesanales asociados en 67 organizaciones gremiales (MAG, 2020). De las 5 grandes caletas establecidas en la provincia, la de Puerto Bolívar resulta la más significativa, en razón que concentra la mayor parte de la población pesquera, los volúmenes de pesca más importantes, la infraestructura básica necesaria y cierta seguridad de operaciones pesqueras por la presencia de la Capitanía del Puerto.

El mar y el comercio de los productos del mismo, han estado relacionados desde tiempos de antaño, representan una alternativa vital para Ecuador. La pesca artesanal sigue siendo uno de los factores determinantes de la economía familiar. Según la Cooperativa de Producción Pesquera Artesanal "Santa Rosa", el sector pesquero artesanal factura 100 millones de dólares al año, lo que significa que es un aporte importante para el país con relación al Producto Interno Bruto (PIB) (Federación Nacional de Cooperativas Pesqueras del Ecuador [FENACOPEC], 2016).

La actividad pesquera artesanal porteña, tiene un peso apreciable en la conformación del
PIB provincial y en la seguridad alimentaria local y regional. Además, genera empleo directo e indirecto y cubre la demanda local así como parte de la demanda nacional respecto al suministro y distribución de productos de mar. La mayor parte del volumen de pesca se comercializa en los mercados de Machala, otro porcentaje se expende en los mercados de Azuay, Loja y Zamora Chinchipe (El Telégrafo, 2014).

El desarrollo de la actividad pesquera artesanal en Puerto Bolívar, se direcciona en dos frentes. En el primero, los pescadores se dedican a la extracción, recolección y comercialización de moluscos (calamar, pulpos, conchas, almejas y mejillones) y crustáceos (cangrejos, langostas y camarones); en el segundo, estos se disponen, con sus pequeñas embarcaciones, a la pesca blanca (corvina, robalo, cachema, bagre, lisa, entre otras especies).

La población inmersa en la pesca artesanal, de manera general, corresponde a estratos sociales pobres y de bajo nivel cultural, que encuentran en esta actividad la posibilidad de obtener ingresos económicos vía captura-comercialización de productos de la pesca para la cobertura de los gastos familiares que demanda la vida cotidiana; pero además, cubre parte de la demanda alimenticia familiar. Si bien es cierto el nivel de ingresos de los pescadores está condicionado a ciertos factores, entre ellos, el estado meteorológico, la suerte de captura, el volumen y calidad de las especies y la dinámica del mercado, sin embargo, el PMRC (2008), da cuenta que el promedio de ingresos oscilaba alrededor de los $\$ 100$ dólares por semana y por pescador. Después de la faena de pesca, el desembarque de los productos de mar se realiza en la misma playa, donde los pescadores artesanales los venden a los comerciantes que están alrededor del sitio.

En estas condiciones, no cabe duda alguna del apreciable aporte del sector pesquero artesanal a la disminución de la pobreza socioeconómica y al mejoramiento de la calidad de vida de los pescadores y sus familias; pero -además- de todo el 
conglomerado humano inserto en las cadenas de procesamiento y comercialización.

\section{Conclusiones}

El recorrido teórico y la observación de las realidades que envuelven la actividad pesquera de carácter artesanal en el Puerto Bolívar, contrastada en modo histórico con las prácticas socioculturales de la zona, permiten elaborar una serie de disquisiciones que pretenden recoger aquellos elementos de tipo cualitativo que definen las interacciones e identidades de la población sobre la que se ha pensado y que representa una suerte de sujeto histórico que encarna, ciertamente lo económico de la actividad; pero -mucho más- la esencia humanística que allí existe. En este segmento se intenta entonces, presentar algunas certezas transitorias encontradas luego del ejercicio de reflexión.

En principio, a pesar de la importancia que encierra la actividad pesquera artesanal en Puerto Bolívar, existe una serie de limitantes que obstaculizan un mayor impacto económico y social. Por ejemplo, muchos pescadores tienen problemas para inculcar en su familia la importancia que la pesquería artesanal tiene para sus vidas. Es evidente, el choque que existe allí entre pertinencia cultural o tradicional e idoneidad económica, pues para muchos jóvenes, la pesquería a ese nivel no les aporta lo necesario para alcanzar la calidad de vida anhelada.

En cuanto al tema organizacional, en Puerto Bolívar hay un importante número de organizaciones que se estructuran alrededor del oficio pesquero en el sector artesanal. Aunque quienes se agrupan allí mantienen los métodos y técnicas propias de la tradición, ciertamente se presentan algunos conflictos con organizaciones de otro perfil, cuya finalidad está ligada a la comercialización del producto. En términos de competitividad, sin duda, la tradición implica algunos obstáculos; sin embargo, se puede apreciar que esta preocupación por el limitante económico que representa este oficio, se mantiene por debajo de lo simbólico cuando se trata de la pesca artesanal.

Llegados a este punto, se considera corroborada la tesis según la cual, para los pescadores artesanales, el lucro económico es importante, pero secundario. Si bien las necesidades de subsistencia les obligan a veces a claudicar frente al mercado y sus métodos, su perfil sociocultural se mantiene. La crianza de los hijos, la conformación de la familia, la axiología que refuerza la relación entre padres e hijos, y entre los géneros, aún mantienen las orientaciones propias de las familias tradicionales de Ecuador.

En el terreno de la familia, es conveniente señalar que en Puerto Bolívar, como en el resto del país, el trabajo infantil y adolescente no existe debido a su prohibición y supervisión permanente; no obstante, puede presentarse de forma clandestina, dependiendo de las características de las familias a la que pertenecen los niños. Al respecto, se puede decir que persisten ciertas manifestaciones de violencia intrafamiliar, en muchos casos, soportadas por esa imagen del poder patriarcal y la figura masculina como centro de la disciplina familiar.

En definitiva, para el diseño y ejecución de políticas públicas y de rediseño de estrategias y acciones de reordenamiento de la actividad pesquera artesanal desde esferas gubernamentales, resulta importante comprender y respetar las prácticas culturales de la población involucrada, hecho que determina la necesidad de que los pescadores sean partícipes de los procesos de planificación, ejecución y evaluación, cuya finalidad debe ser el fortalecimiento de las comunidades pesqueras artesanales como estrategia de seguridad alimentaria, coadyuvando a la vez al mejoramiento de la calidad de vida de esta población.

En congruencia con este último aspecto, debe insistirse en corregir los efectos perniciosos que sobre el desarrollo de la pesca artesanal han tenido las actividades llevadas a cabo por los intermediarios y los grandes pesqueros, quienes no solo afectan desde el punto de vista económico, sino que producen 
Fernández-Espinosa, Cira; Brito-Paredes, Patricio; Mendoza-Torres, Galo y VillavicencioAguilar, Carmita

Tradición pesquera artesanal e identidad sociocultural de Puerto Bolivar: Contexto del Golfo de Guayaquil-Ecuador

también una afectación cultural al intervenir en los procesos socioculturales que se tejen a lo largo de la cotidinidad de los pescadores y sus familias, provocando, en algunos casos, procesos de transculturización no deseables para el sostenimiento de las tradiciones.

Así mismo, el tema ecológico es importante considerarlo entre los aspectos que pemitirían construir políticas públicas acertadas para el sector pesquero artesanal, al tiempo que se cumple con el tradicional respeto que el pescador artesanal siente por la naturaleza. Está claro, que si no se realiza un uso racional de los recursos naturales, especialmente de los manglares y se detiene su tala indiscriminada para la construcción de las camaroneras (actividad que pudiese tener un sentido económico comprensible), provocará -sin duda- la merma paulatina de los recursos y la desparición del sustento de muchas familias que viven de este muy maltratado ecosistema.

\section{Referencias bibliográficas}

Aguilar, F. E. (2013). Puerto Bolivar, realidad y prospectiva en el ámbito turístico (Tesis de maestría). Universidad del Azuay, Cuenca, Ecuador.

Alvarado, N. (2016). Debate internacional sobre pobreza. Revista de Ciencias Sociales (Ve), XXII(3), 104-121.

Álvarez, M. C., Ruiz, G., Collao, D., y Gajardo, C. (2017). La visualización femenina en la pesca artesanal: Transformaciones culturales en el sur de Chile. Polis, 16(46), 175-191. http://dx.doi.org/10.4067/S0718$\underline{65682017000100175}$

Álvarez, R., Núñez, L., Calderón, F., y Mendoza, E. (2020). Producción y comercialización de productos de curtiembre de piel de pescado, Santa Elena - Ecuador. Revista de Ciencias Sociales (Ve), XXVI(4), 353367. https://doi.org/10.31876/rcs. v26i4.34667
Anaya, A. R., Buelvas, J. A., y Romero, Y. (2020). Pobreza e inclusión financiera en el municipio de Montería, Colombia. Revista de Ciencias Sociales (Ve), XXVI(1), 128-143. https://doi. org/10.31876/rcs.v26i1.31315

Banchón, A. B., Vite, H., y Carvajal, H. (2020). Perfil socioeconómico y ambiental de la pesca artesanal en la comuna riveras de Huayla. Revista Metropolitana de Ciencias Aplicadas, 3(2), 199-205.

El Comercio (27 de Junio de 2009). El pescador hereda el oficio de sus abuelos. El Comercio. https://www. elcomercio.com/actualidad/pescadorhereda-oficio-abuelos.html

El Comercio (15 de agosto de 2018). Autoridades confirma que desmanes en Puerto Bolívar no fueron causados por pescadores artesanales. El Comercio. https://www.elcomercio. com/actualidad/autoridades-confirmadesmanes-puertobolivar-pescadores. $\underline{\mathrm{html}}$

El Telegráfo (16 de noviembre de 2014). La pesca es el motor de desarrollo de Puerto Bolivar: La mayoría de los mariscos se dirigen a Cuenca, Loja y Zamora Chimchipe. El Telégrafo. https://www.eltelegrafo.com.ec/ noticias/regional-sur/1/la-pesca-es-el$\underline{\text { motor-de-desarrollo-de-puerto-bolivar }}$

El Universo (5 de marzo de 2018). Mariscos, parte del atractivo turístico de Puerto Bolívar. El Universo. https://www. eluniverso.com/noticias/2018/03/05/ nota/6651841/mariscos-parteatractivo-turistico-puerto-bolivar

Federación Nacional de Cooperativas Pesqueras del Ecuador - FENACOPEC (2016). Cooperativa de Producción Pesquera Artesanal "Santa Rosa". FENACOPEC.

Gobierno Autónomo Descentralizado (GAD) Municipal de Machala (2018). Plan de 
Desarrollo y Ordenamiento Territorial de Machala (PDyOT_2018 Machala). GAD Municial de Machala. https:// www.machala.gob.ec/features/ plan-de-desarrollo-y-ordenamientoterritorial/

González-Garcés,A. (2006). La sustentabilidad de los recursos pesqueros en términos biológicos. Revista Galega de Economía, 15(1), 1-21.

Herdson, D., Rodríguez, W., y Martínez, J. (1985). Las pesquerías artesanales de la costa del Ecuador y su captura en el año 1982. Instituto Nacional de Pesca. https://www.oceandocs.org/ handle/1834/3225

Herrera, M., Castro, R., Coello, D., Saa, I., y Elías, E. (2013). Puertos, Caletas y Asentamientos pesqueros artesanales en la csta continental del Ecuador. Instituto Nacional de Pesca (INP).

Instituto Nacional de Pesca (15 de noviembre de 2019). La Asociación de Mujeres Artesanas Estero Porteño elabora artesanías con escamas, conchas y basura plástica. Instituto Nacional de Pesca. http://www.institutopesca.gob. ec/artesanas-cerro-porteno-elaboraartesanias-escamas-conchas-basuraplastica/

Kurien, J. (23 de Noviembre de 2020). Los aspectos socioculturales de la pesca: Repercusiones en la seguridad alimentaria y de los medios de subsistencia. Estudio de caso del estado de Kerala, India. FAO. http:// www.fao.org/3/y1290s/y1290s11.pdf

Leante, D., y García, L. A. (2012). "Proyecto para el desarrollo de las comunidades pesqueras de la República del Ecuador": Proyecto 082. Cuaderno 1 Memorias del Proyecto. Universidad Politécnica de Madrid. Escuela Técnica Superior de Ingenieros Navales. http://oa.upm.es/14340/1/ PFC Daniel_Leante Darricau.pdf
León-Valle, W., Núñez-Guale, L., Valencia , A., y Cedeño, J. (2017). La Pesca Artesanal un legado del saber ancestral, provincia de Santa Elena. Revisa de Investigaciones Sociales, 3(10), 51-63.

McGoodwin, J. R. (2002). Comprender las culturas de las comunidades pesqueras: Clave para la ordenación pesquera y la seguridad alimentaria. Organización de las Naciones Unidas para la Agricultura y la Alimentación (FAO). http://www.fao.org/3/y1290s/ y1290s.pdf

Méndez, F. M. (2020). Territorialidades en tensión: el caso de los pescadores artesanales en Delta del Paraná (20122017). Revista Huellas, 24(1), $195-$ 215.

Ministerio de Acuacultura y Pesca (5 de marzo de 2017). Predio camaronero fue concesionado a pescadores artesanales del Golfo de Guayaquil. Ministerio de Acuacultura y Pesca. http://acuaculturaypesca.gob.ec/ subpesca3062-predio-camaronerofue-concesionado-a-pescadoresartesanales-del-golfo-de-guayaquil. $\underline{\mathrm{html}}$

Ministerio de Ambiente y Agua (26 de julio de 2016). Nuevos Guardianes del Manglar reciben 900 hectáreas, tras firma de Acuerdo en el Golfo de Guayaquil. Ministerio de Agricultura $y$ Agua. https://www.ambiente.gob. ec/nuevos-guardianes-del-manglarreciben-900-hectareas-tras-firma-deacuerdo-en-el-golfo-de-guayaquil/

Ministerio de Agricultura y Ganadería - MAG (2020). Pescadores artesanales, de El Oro, reciben facilidad pesquera. Ministerio de Agricultura $y$ Ganaderia. https://www.agricultura. gob.ec/pescadores-artesanales-de-eloro-reciben-facilidad-pesquera/

Organización de las Naciones Unidas para 
Fernández-Espinosa, Cira; Brito-Paredes, Patricio; Mendoza-Torres, Galo y VillavicencioAguilar, Carmita

Tradición pesquera artesanal e identidad sociocultural de Puerto Bolivar: Contexto del Golfo de Guayaquil-Ecuador

la Agricultura y la Alimentación - FAO (2003). Estudio sobre el impacto socioeconómico de la pesca artesanal en los Estados miembros de la Comisión Permanente del Pacifico Sur. FAO. http://cpps.dyndns.info/ cpps-docs-web/planaccion/biblioteca/ pordinario/031.EstudioImpactodelaPe scaArtesanalenPaisesMiembrosdelaC PPSversion203.pdf

Organización de las Naciones Unidas para la Alimentación y la Agricultura FAO (2019). Papel de la FAO en la acuicultura. FAO. http://www.fao.org/ aquaculture/es

Paredes, J. (2000). Aprovechamiento de la riqueza marino costera como alternativa para el desarrollo nacional (Tesis de maestría). Instituto de Altos Estudios Nacionales, Quito, Ecuador.

Programa de Manejo de Recursos Costeros - PMRC (1993). Plan de Manejo de la ZEM Machala - Puerto Bolivar - Jambeli. Programa de Manejo de Recursos Costeros. https://www.crc. uri.edu/download/Machala ZEM Spanish.pdf

Programa de Manejo de Recursos Costeros - PMRC (2008). Procesamiento de información: Censos Costeros Pesqueros Atesanales. Provincia Manabi. PMRC-2008. http://oa.upm. es/id/file/257779

Working to sustain the natural world for the benefit of people and nature WWF-Laos (29 de agosto de 2016). Pescadores artesanales proponen una pesca responsible y ordenada de camarón pomada dentro del Golfo de Guayaquil. WWF-Laos. https://www.wwf.org.ec/?276670/ Pescadores-artesanales-proponen-unapesca-responsable-y-ordenada-decamarn-pomada-dentro-del-Golfo-deGuayaquil 